\title{
Shifts in magnitude of delayed and immediate reward
}

\author{
W. MILES COX \\ University of Oregon Health Sciences Center, Portland, Oregon 97201 \\ and \\ ROGER W. BLACK \\ University of South Carolina, Columbia, South Carolina 29208
}

\begin{abstract}
In a 2 by 2 factorial design, 48 albino rats were trained in a straight runway to receive either a large or a small magnitude of immediate or delayed reward, after which all groups received the large reward for an additional phase of training. The groups receiving immediate reinforcement performed more rapidly than the delayed groups throughout the experiment. The effects for reward magnitude were complex, and there was some suggestion that delayed reinforcement produced a reversal of the usual superior performance for a large reward. While delayed upshifted subjects briefly overshot the performance level of large-reward controls, group differences were not reliable, and this failure to obtain a positive contrast effect did not seem to be due to a physiological limit.
\end{abstract}

Traditionally, it has been found that animals which are trained with one magnitude of reward and then are shifted to a different magnitude show abrupt changes in performance, and, in addition, negative "contrast effects" (NCEs) are obtained in that subjects which are shifted from a larger to a smaller magnitude respond more slowly than subjects which have always received that smaller reward. On the other hand, positive "contrast effects" (PCEs) do not occur since upshifted subjects fail to respond more rapidly than subjects which have been trained consistently with the larger reward (Black, 1968; Dunham, 1968). Recently, however, the conclusion that incentive contrast effects are asymmetrical-i.e., that PCEs are not obtained-has been challenged by experimenters who assert that PCEs which otherwise would have appeared have simply been obscured through limitations of the experimental training procedure. It is argued, that is, that large-reward control subjects come to run at maximum speeds and reach a physiological limit so that it is physically impossible for upshifted subjects to respond more rapidly (e.g., Mellgren, 1972).

In an attempt to circumvent this difficulty, delayed reinforcement has been employed in order to lower asymptotic running speeds and thus make it possible for upshifted subjects to exceed the level of performance of large-reward controls. Although PCEs have been reported in every study of "successive" upshifts in magnitude of delayed reinforcement, these studies have been few in number; reliable group differences in some cases have failed to occur (Mellgren, 1971); and in other cases, the "overshooting" of upshifted subjects has appeared for no more than one trial block of the postshift phase (Mellgren, 1972, Experiment I). Thus, the evidence at this point is not entirely convincing that

The authors wish to thank Karen Hall and Judy Lawson for their assistance in running the subjects. the PCE is reliable with this procedure. While, moreover, both positive (Mellgren, Wrather, \& Dyck, 1972) and negative (Chechile \& Fowler, 1973) results have been obtained in tests for PCEs in differential conditioning procedures employing "simultaneous" shifts in magnitude of delayed reward, the evidence is overwhelmingly negative in cases of simultaneous (Chechile \& Fowler, 1973; Gavelek \& McHose, 1970) and successive (McHose \& Tauber, 1972; Shanab \& McCuistion, 1970; Shanab, Rouse, \& Cavallaro, 1973) shifts in the length of delay under a constant magnitude of reward, even though in these latter situations delayed reinforcement also reduces asymptotic levels of performance and thus should make it possible for PCEs to be demonstrated. Due to this ambiguity and sparcity of present empirical evidence, additional data are needed to clarify both the specific circumstances which give rise to the PCE under delayed reinforcement and to indicate whether the physiological limit is a correct explanation to account for the consistent absence of PCEs with immediate reinforcement, and the present study provides additional evidence in this regard.

\section{METHOD}

\section{Subjects}

The subjects were 48 albino rats of the Sprague-Dawley strain, 120-130 days old at the beginning of training; which were recruited from the colony maintained by the Department of Psychology at the University of South Carolina.

\section{Apparatus}

The apparatus consisted of a standard straight runway whose dimensions corresponded with those of a runway previously employed (Mellgren, 1971). The startbox and alley were $31 \mathrm{~cm}$ and $121.9 \mathrm{~cm}$ long, respectively, and each was $13 \mathrm{~cm}$ wide, while the goalbox measured $20 \mathrm{~cm}$ in width and $33 \mathrm{~cm}$ in length. All sections were $21 \mathrm{~cm}$ high. Transparent doors separated the startbox and goalbox from the alley, and a third door, painted flat black and located $6 \mathrm{~cm}$ from the end wall of the goalbox, separated the goal dish from the remainder of the goalbox. The 
goal cup consisted of a measuring teaspoon which was mounted at the back wall of the goalbox, $5 \mathrm{~cm}$ from the floor. Response times were measured with photoelectronic circuitry and were divided into three segments: start time, which was measured from the opening of the startbox door to a point $15.24 \mathrm{~cm}$ inside the alley; run time, which was measured over the next $91.42 \mathrm{~cm}$; and goal time, which was measured from a point $15.24 \mathrm{~cm}$ from the goalbox door to a point $5.08 \mathrm{~cm}$ inside the goalbox, or a total distance of $20.32 \mathrm{~cm}$.

\section{Procedure}

Phase I of the experiment consisted of a 2 by 2 factorial design in which delay of reinforcement (delayed or immediate) and magnitude of reward (small or large) were varied, with the resulting groups being designated large-immediate, small-immediate, large-delayed, and small-delayed. Twelve subjects were assigned to each group under the stipulation that the groups be matched on the basis of age and weight of the animals. Ten days before training was initiated, the subjects were placed on a food deprivation regimen which consisted of the daily administration of $9 \mathrm{~g}$ of lab chow, a procedure which was continued throughout the experiment except that appropriate subtractions were made for food received in the goalbox after the initiation of training. On 3 days of the initial deprivation period, subjects were handled individually for approximately 5 min each, and, on the day immediately preceding acquisition, subjects explored the apparatus in groups of two animals each for $5 \mathrm{~min}$.

Two experimenters each ran two squads of 12 subjects each, with four subjects from each of the four groups being assigned to a squad in a random sequence. On the 1 st day of training, each subject was administered one trial; on Days 2 and 3, two trials; on Day 4, three trials; and four trials on each of the remaining 21 days, for a total of 104 trials for Phase I. A trial was initiated by placing the subject into the startbox facing the startbox door. After a 4-sec interval, the startbox door opened automatically and then reclosed when the subject interrupted the first photobeam, and when the subject had entered the goalbox, the goalbox door was reclosed manually to prevent reentry of the alley. For the immediate groups, the door which concealed the goal cup opened immediately and automatically when the subject entered the goalbox; while for the delayed groups, the door remained closed for $20 \mathrm{sec}$ after subject's entry into the goalbox. Reward consisted of six and one 45-mg Noyes food pellets for the large-reward and small-reward groups, respectively. As soon as the subject had consumed its reward, it was returned to the carrying cage to await its next trial, with the resulting intertrial interval being approximately $15-20 \mathrm{~min}$. As soon as a squad's trials were completed, the animals were returned to their home cages and fed their maintenance diet.

On the day following completion of Phase I, Phase II was initiated, during which four daily trials were administered for 6 days. During this phase, all groups received the large (six pellets) reward, but all groups continued to receive the same delay procedure that they had received during Phase I.

\section{RESULTS AND DISCUSSION}

Prior to analysis, all response latencies were converted into speeds (feet per second). Since separate graphical representation of the data collected by the two experimenters indicated no systematic differences, the data were collapsed before additional analyses were made. ${ }^{1}$ Since start, run, and goal speeds yield some what different information, all three measures are included in the present discussion.

\section{Phase I}

Initial inspection of the results of Phase I, shown in Figure 1, indicates that for all three measures immediate reward led to faster performance than delayed reward, $\mathrm{F}(1,38)=20.57, \mathrm{p}<.001 ; 4.16, \mathrm{p}<.05 ;$ and 31.48 , $\mathrm{p}<.001$, respectively. There also were changes in performance with trials, $F(25,950)=57.52, p<.001$; $36.53, \mathrm{p}<.001$, and 12.57, $\mathrm{p}<.001$, respectively, but the initial improvement was more precipitous for the immediate than for the delayed groups, especially in the case of goal speeds, and this difference is reflected by statistically reliable Delay by Trial interactions, $\mathrm{F}(25,950)=4.01, \mathrm{p}<.001 ; 3.21, \mathrm{p}<.001 ; 6.34$, $\mathrm{v}<.001$, respectively.

Less consistent and more complex effects, on the other hand, were produced by the two magnitudes of reward. Thus, in the case of both run and goal speeds, subjects trained under immediate reinforcement performed more rapidly for the large reward than for the small reward during initial training, but this difference had diminished or disappeared by the end of the preshift phase, and although reward magnitude was not a significant source of variance in performance during Phase I as a whole, the initial superiority of large-reward over small-reward subjects trained under immediate reinforcement (i.e., during the first 10 trial blocks of Phase I) was reliable for goal speeds, $F(1,18)=4.51$, $\mathrm{p}<.05$, and approached significance for run speeds, $\mathrm{F}(1,18)=3.22, .05<\mathrm{p}<.10$. For start speeds, on the other hand, the superiority of the large-immediate over the small-immediate group is apparent throughout Phase I, and, since delayed subjects did not respond differently for large and small reward, the former effect is reflected by a reliable Delay by Reward interaction, $\mathrm{F}(1,38)=4.85, \mathrm{p}<.05$.

For run and goal speeds as well there is no indication that large reward produced superior performance among the delayed subjects. On the contrary, there is some indication of a reversal of the usual magnitude-of-reward effect (viz., the initial one-third of Phase I for running speeds and the latter one-thrid for goal speeds). While in the former case the difference is not statistically reliable, $F(1,20)=2.26, \quad p>.10$, the latter approaches significance, $F(1,18)=3.74, .05<p<.10$. In short, while the results from Phase I indicate differences in performance produced by magnitude of reward, these differences were neither permanent nor consistent. In this sense, the present data agree with other recent findings which question the traditional view that subjects consistently perform more rapidly for a large than for a small reward (Black, 1969; McCain, Dyleski, \& McElvain, 1971). The present results imply, in addition, that a reversal of the customary effect might be more likely to appear with delayed than with immediate reinforcement. 


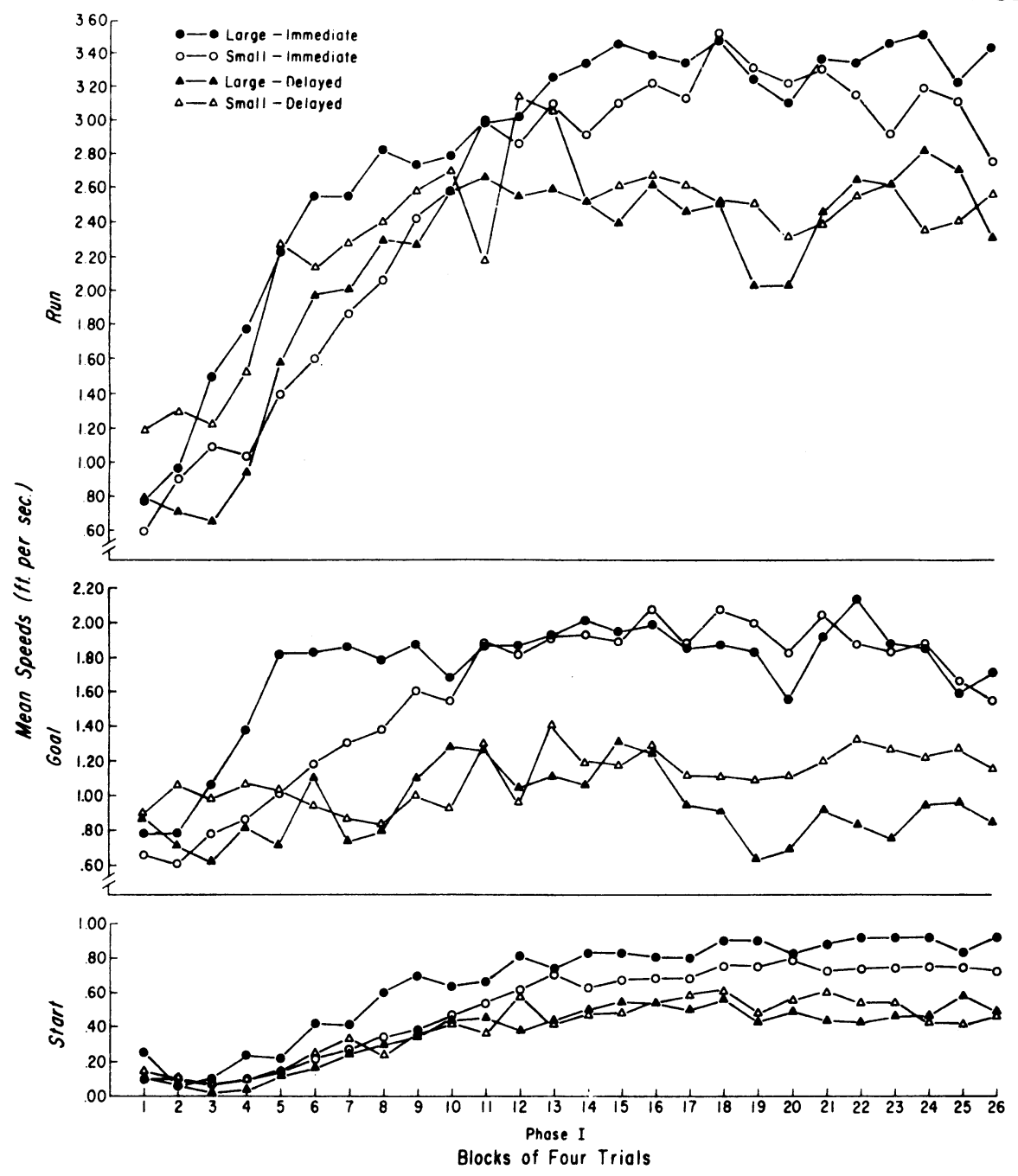

Figure 1. Mean speeds during Phase I for the four groups as a function of blocks of four trials.

\section{Phase II}

Start, run, and goal speeds for the 24 trials of Phase II, along with the last 24 trials of Phase I, are presented in Figure 2. Considering first the groups trained under immediate reinforcement, initial inspection provides some indication that for both run speeds (Trial Blocks 24) and goal speeds (Trial Blocks 1-4), but not for start speeds, upshifted subjects ran more rapidly during Phase II than subjects which had always been trained under the large reward. Statistical analysis of these trial blocks indicates, however, that in neither case does the superiority of upshifted subjects approach significance, $F<1.0$, and $F(1,18)=1.98$, $\mathrm{p}>.10$, respectively.

Considering next the groups trained under delayed reinforcement, start speeds indicate a small initial superiority (Trial Blocks 1-3) for upshifted subjects similar to that which occurred for the immediate groups, and statistical analysis indicates that in this case the groups also did not reliably differ
$(\mathrm{F}=1.0)$. The possibility that the preceding differences in upshifted and control groups reflects a PCE is further mitigated by the fact that similar differences had already occurred during the preshift phase (e.g., Trial Blocks 16-21 for goal speeds, immediate groups; $17-23$ for start speeds, delayed groups). Goal speeds for the delayed groups suggest that although the small-reward group was responding more rapidly than the large-reward group at the conclusion of preshift training, there were further increases in speeds during Phase II. However, the interaction of groups with preshift-postshift performance (during the six postshift trial blocks and the last six preshift trial blocks), which would have reflected this additional increase, yielded an $F$ ratio which was less than 1.0. Finally, the strongest indication of a PCE is shown by the running speeds of the delayed groups where not only was there an abrupt increment in performance with the upshift in reward, but, graphically, there appears to be a decided "overshooting" during the first three trial blocks of the postshift phase. Even in this 

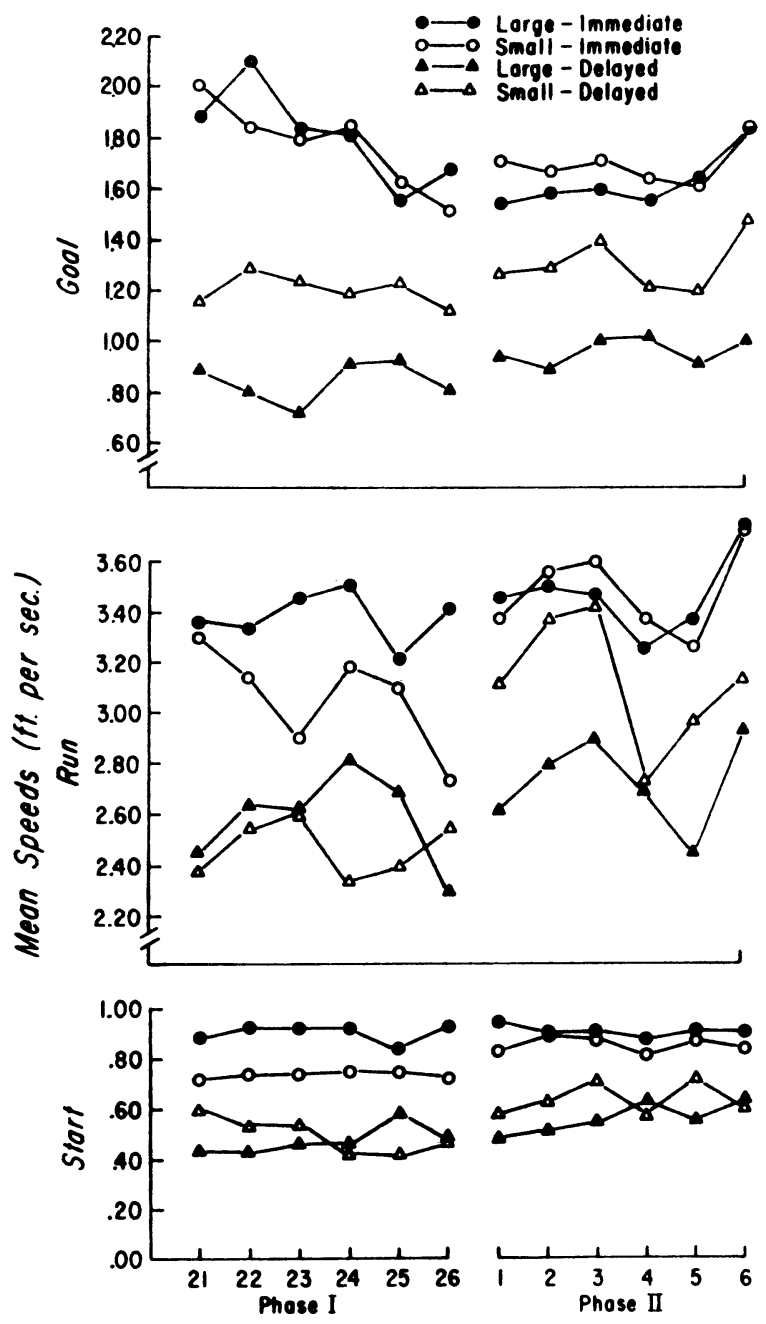

Blocks of Four Triols

Figure 2. Mean speeds during Phase II and the terminal trials of Phase I for the four groups as a function of blocks of four trials. Groups are designated according to the magnitude of reward and delay conditions under which they were trained during Phase I.

case, however, a reliable difference between the upshifted and control group was not obtained, $\mathrm{F}(1,18)=1.81, \mathrm{p}>.10$.

The lack of evidence for PCEs for the immediate groups during Phase II is consistent with previous studies which have failed to obtain PCEs with immediate reinforcement. The present results, however, which also provide no reliable evidence for PCEs with delayed reinforcement, do not support the results of previous tests for successive PCEs with this procedure. It should be noted, moreover, both (1) that the performance of the delayed groups was reliably suppressed relative to that of the immediate groups and (2) that the immediate groups, at least in the case of goal speeds, had responded more rapidly during a substantial portion of Phase I than they ever responded during Phase II, and thus it appears that it would have been physically possible for the upshifted subjects which were trained with both procedures to have responded more rapidly during Phase II. Nevertheless, the overshooting which was observed in the running speeds of the upshifted delayed group, even though it was not reliable, resembles the rapidly disappearing "positive contrast" which was previously reported.(Mellgren, 1971, 1972), and thus the possibility remains that some mechanism which is peculiar to delayed procedures is responsible for this similarity of outcomes. While we are strongly dissuaded from main taining that such overshooting results from an elimination of a physiological limit, it appears that firm conclusions regarding the authenticity of "positive contrast" with successive upshifts in magnitude of delayed reward must await additional empirical evidence.

\section{REFERENCES}

Black, R. W. Shifts in magnitude of reward and contrast effects in instrumental and selective learning: A reinterpretation. Psychological Review, 1968, 75, 114-126.

Black $R$. W . Incentive motivation and the parameters of reward in instrumental appetitive conditioning. In W. Arnold (Ed.), The Nebraska symposium on motivation. Lincoln: University of Nebraska Press, 1969.

Chechile, R., \& Fowler, H. Primary and secondary negative incentive contrast in differential conditioning. Journal of Experimental Psy chology, 1973, 97, 189-197.

Dunham, P. J. Contrasted conditions of reinforcement: A selected critique. Psy chological Bulletin, 1968, 69, 295-315.

Gavelek, J. R , \& McHose, J. H. Contrast effects in differential delay of reward conditioning. Journal of Experimental Psy chology, 1970, 86, 454-457.

McCain, G., Dyleski, K., \& McElvain, G. Reward magnitude and instrumental responses: Consistent reward. Psychonomic Science Monograph Supplements, 1971, 3, No. 16 (Whole No. 48), 249-256.

McHose, J. H., \& Tauber, L. Changes in delay of reinforcement in simple instrumental conditioning. Psychonomic Science, 1972, 27, 291-292.

Mellgren, R. L. Positive contrast in the rat as a function of number of preshift trials in the runway. Journal of Comparative and Phy siological Psychology, 1971, 77,

329-336.
Mellgren, R. L. Positive and negative contrast effects using delayed reinforcement. Learning and Motivation, 1972, 3, 185-193.

Mellgren, $\dot{R}$. L., Wrather, D. M., \& Dyck, D. G. Differential conditioning and contrast effects in rats. Journal of Comparative and Physiological Psychology, 1972, 80 , 478-483.

Shanab, M. E., \& McCuistion, S. Effects of shifts in magnitude and delay of reward upon runway performance in the rat. Psychonomic Science, 1970, 21, 264-266.

Shanab, M. E. Rouse, L. O., \& Cavallaro, G. Effects of shifts in delay of reward in rats as a function of reward magnitude. The Journal of General Psychology, 1973, 89, 59-66.

\section{NOTE}

1. The data of several subjects which died during the experiment were eliminated.

(Received for publication March 24, 1975.) 